\title{
DESIGN, SUVENIR E CULTURA: ABRANGÊNCIAS DA EXPERIÊNCIA TURÍSTICA
}

Ana Carolina de Moraes Andrade Barbosa

Universidade Federal de Campina Grande (UFCG)

anacarolinamab@gmail.com

Virgínia Pereira Cavalcanti

Universidade Federal de Pernambuco (UFPE)

cavalcanti.virginia@gmail.com

Resumo: Este ensaio discute a relação que um forasteiro estabelece com o espaço turístico e os artefatos que se propõem a transportar a memória da experiência vivida, delimitados nesta ocasião como os suvenires. Para isto, buscou-se apreender os valores identitários construídos do lugar na memória coletiva, através dos monumentos como materialização da história e elementos urbanos de significação social. Foi traçada uma análise entre conceitos contemporâneos que contextualizam esse tema, sendo eles históricos, morfológicos e significativos relacionados aos espaços turísticos, à cultura territorial e ao suvenir. O resultado desta busca sistemática pelo conhecimento ampliado das evidências existentes permitiu o debate também de assuntos complementares como produtos de terroir, memória coletiva do lugar, design e seu caráter globalizado e integrador das abordagens.

Palavras-chave: cultura, território, turismo, suvenir e design.

Abstract: This study discusses the relationship that a tourist has with the space and the artifacts they propose to carry the memory of lived experience, delimited on this occasion as souvenirs. For this, it drew a analysis of concepts that contextualize this subject, they are historical, morphological and significant related to tourist areas, territorial culture and souvenir. The result of this systematic search for knowledge expanded the existing evidence, also allowed the discussion of further issues such as terroir products, collective memory of the place, design and its global and inclusive character of the approaches.

Keywords: culture, territory, tourism, souvenir and design. 


\section{INTRODUÇÃO}

Desde o final do século $X X$, as sociedades globalizadas, tendem, cada vez mais, fragmentar a rigidez das paisagens culturais de classe, gênero, sexualidade, etnia, raça e nacionalidade, que, no passado, nos tinham fornecido personalidade como integrantes de grupos sociais. Nesta perspectiva, essa mudança não se refere apenas às condições geográficas da urbanização das cidades, mas a um novo comportamento das sociedades atuais que estão em constante mudança, rápida e permanente.

Nas sociedades contemporâneas, somos confrontados por uma gama de diferentes identidades, dentre as quais parece complexo fazer uma escolha. O sujeito, previamente vivido como tendo uma identidade unificada e estável, está se tornando fragmentado; composto não de uma, mas de várias identidades. A capacidade de relacionar o que já se viveu ou aprendeu com a situação presente é um mecanismo de constituição e preservação da identidade de cada um. Para Hall (1996), existe uma relação de interdependência entre a vida social e o mercado global, mediada por estilos, lugares e imagens; pelas viagens internacionais; pelas imagens da mídia e pelos sistemas de comunicação globalmente interligados. Quanto mais dependente deste mercado, mais as identidades se tornam desvinculadas de tempos, lugares, histórias e tradições específicas e parecem estar disponíveis para um processo de seleção.

Este processo de identidade cultural e aculturação ${ }^{1}$ tem como foco, para este artigo, o turismo. Pretende-se discutir aqui a relação que um forasteiro estabelece com o espaço turístico e os artefatos que se propõem a transportar a memória da experiência vivida. Com essa questão, já consideramos a primeira hipótese de que existam artefatos com essa função, delimitados nesta ocasião como os suvenires. Para isto, foi traçada uma análise entre conceitos que contextualizam esse tema, sendo eles morfológicos e significativos relacionados aos espaços turísticos, à cultura territorial e ao suvenir. Assim como ao design e seu caráter globalizado.

Vale ressaltar, previamente, que o objeto de estudo deste levantamento não se centra no artefato ou no espaço turístico, e sim no sujeito. Em questão, refere-se ao flaneur (ou o caminhante), que vagueia entre as novas arcadas das lojas, observando o passageiro espetáculo da metrópole, que Walter Benjamin celebrou no seu ensaio sobre a Paris de Baudelaire, e cuja contrapartida na modernidade tardia é, provavelmente, o turista (HALL; 1996). É um observador em movimento, em busca de viver situações inesperadas, disposto a gastar tempo e/ou dinheiro com momentos diferentes do que estão acostumados, os passeios turísticos. E, por fim, ter a altivez de voltar para sua rotina, carregado de novas imagens e produtos que relatam o conhecimento de uma nova cultura e a presenteiam como prova de estima do que viveram.

O sumário dos conceitos acadêmicos, que cercam o tema na contemporaneidade, estão estruturados neste documento em tópicos. Inicialmente, buscou-se compreender a complexidade da cultura territorial e o seu reflexo no espaço turístico. Em seguida, o produto de terroir, seu caráter identitário e o Design como ferramenta estratégica de interseção entre as áreas. Por fim, o suvenir e a materialização comercial da memória.

\footnotetext{
${ }^{1}$ Segundo Santaella (2003), significa a troca de elementos culturais por dois grupos sociais quando são postos em contato.
} 


\section{CULTURA E TERRITÓRIO}

O conceito do termo cultura, no sentido original da palavra, significa o ato de cultivar o solo. Com o passar dos séculos, perdeu seu sentido etimológico e passou a compreender as particularidades que formam a enorme diversidade da humanidade. Para Katan (2012), a cultura está fortemente relacionada com o turismo; os termos são inseparáveis quando se trata de ministérios e secretarias municipais, por exemplo. 0 autor utiliza a expressão "cultura turística" para designar situações cotidianas de habitantes ou, extraordinárias, de visitantes em territórios repletos de hotéis, restaurantes, pontos turísticos, e etc. Para os turistas, esse universo modifica também o estilo de se vestir, os acessórios (mochila, água, câmera, mapa, etc.); a linguagem; e o comportamento (mudança no tipo de transporte, a observação curiosa do espaço e dos habitantes, etc.).

Neste contexto, temos o que Katan (2012) chama de o "turista cultural", aquele que viaja com a intenção de reunir novas informações e experiências para satisfazer necessidades culturais. Um ponto de vista interessante sobre o conceito é o antropológico abordado pelo autor, pois trata-se de um sistema de concepções inerente e compartilhado por um grupo. O modelo de perceber e interpretar as coisas que cria a noção do que é normal para uma cultura. Esse padrão de normalidade é transmitido entre gerações que determinam valores e crenças. A preservação dessa propriedade tradicional é atribuída à ideia do patrimônio.

Neste contexto, o turismo de patrimônio cultural significa não só viajar para experimentar os lugares e artefatos, mas também experimentar as atividades que representam autenticamente histórias e pessoas do passado e do presente (KATAN; 2012). O patrimônio significa pertencimento à civilização, ou seja, à cultura, e pode ser materializado por meio dos espaços turísticos formados por monumentos, grupos de edifícios e paisagens naturais e/ou do homem.

\subsection{Espaços turísticos}

Compreendemos que estudar espaços reconhecidos como turísticos envolve uma noção de identificação não apenas espacial, arquitetônica ou urbanística. Essa identificação que nos referirmos está historicamente, e não biologicamente, inerente a uma nação. Um sentimento de reconhecimento nacional de lealdade e dever de representação cultural de um lugar. O sentimento local, quando confrontado aos nacionalismos do mundo, buscam expressar algum tipo de particularismo que enriquece e caracteriza suas experiências culturais como únicas por terem sido inseridas naquele entorno específico.

Osborne (2001) reforça a ideia de que a identificação das pessoas com determinados locais é essencial para o cultivo de uma consciência da identidade nacional. Elas são geradas por processos simbólicos que emergem e se dissolvem em contextos particulares de ação. Desta forma, os espaços tornam-se repletos de lugares "simbolicamente carregados" e eventos que fornecem continuidade social, "contribuem para a memória coletiva, e estabelecem pontos de referência espacial e temporal para a sociedade". Há uma relação de reciprocidade em curso entre as pessoas e os lugares. "As pessoas produzem lugares, e ainda assim derivam identidades deles". Para essa afirmação, Osborne (2001) se reforça citando McDowell (1997): "as pessoas são constituídas através de lugares". 
A noção de nação requer um senso de consciência e identidade coletiva que é promovida através de um sentimento comum de experiência histórica. Para este fim, Osborne (2001) acredita que o uso criativo de símbolos e mitos, monumentos e comemorações nutrem e reforçam algum tipo de identidade e herança dela.

Os espaços carregados de história e identidades são tratados por Osborne (2001) como paisagens (termo que segundo o autor é utilizado na geografia para esse fim): "as paisagens servem muitas vezes como veículos emocionais através do que Halbwachs chamou de semiótica do espaço" (OSBORNE, 2001). Percebemos, então, que existe uma relação hierárquica na identificação ou concepção de um espaço turístico para que ele vire interesse de um forasteiro; para tal, é preciso que antes seja contemplado, aprendido, vivenciado por um habitante, de gerações atuais ou passadas. Mais especificamente, que o lugar faça parte da tradição herdada da identidade territorial de um povo. Só assim ele poderá gerar também experiências e memórias que se perpetuem para visitantes de culturas diferentes.

\section{O PRODUTO TERROIR}

Aurier, Fort e Sirieix (2005) identificam uma nova estratégia de diferenciação mercadológica relacionada à exploração de um território e aplicada ao comércio. 0 experimento é direcionado aos produtos alimentícios; eles justificam essa categoria como relevante para estudar o conceito de terroir, devido à sua ligação natural e forte com a cultura, além das características geológicas e sociológicas. A pesquisa possui caráter exploratório e consiste em entrevistas qualitativas, seguida por uma análise quantitativa. $\mathrm{O}$ estudo qualitativo envolveu 12 entrevistas em três grupos focais. A análise do material gerou um conjunto de variáveis relevantes que foram utilizados para desenvolver um questionário para amostra de 53 indivíduos consumidores.

O resultado do experimento direcionou a compreensão do produto terroir, de acordo com os consumidores, para um artefato ligado à região, ao território e à terra. Comprar um produto terroir é como adquirir a imagem do lugar, não só a qualidade, uma espécie de escapismo a um contexto familiar, ou apenas para outro lugar (AURIER, FORT e SIRIEIX; 2005). Essa dimensão afetiva agrega aos produtos terroir tradição e idealização territorial.

A região de origem pode ser indicada na embalagem alimentícia como uma informação intrínseca assim como preço, quantidade, etc. Mas, tem sido considerado também como uma categoria cognitiva que atribui como qualidade a ideia de que um produto é mais típico do que outros.

Numa visão mais radical de Aurier, Fort e Sirieix (2005), "assim que um produto é industrializado, ele não é mais um produto de terroir"; o fator de diferenciação atribuído ao processo de fabricação é percebido como uma garantia da sua autenticidade. Os autores concluíram, ainda na pesquisa exploratória, que o envolvimento com produtos terroir está relacionado especialmente com tempo e cultura. Para o autor, esse resultado indica que o maior incentivo para o consumo é a dimensão emocional e simbólica de terroir. Trata-se da transferência de valores culturais de um lugar para um artefato.

Neste contexto de comércio, turismo, cultura e autenticidade, verifica-se que 
existem temáticas, como a pesquisa do suvenir, que absorve todo o teor complexo discutido anteriormente. Horodyski et al (2013) estuda a abordagem existente sobre o suvenir e afirma que o tema é tratado como pejorativo e sem legitimidade acadêmica; com base nisso, a importância do tema é rebatido, ressaltando o valor simbólico e do marketing de destinos turísticos que o Mercado de souvenir promove. Em parte, porque esse objeto é considerado banal, de baixo custo e consumido massivamente pelos visitantes; e, embora seja bastante comum encontrar ao menos um souvenir na casa da maioria das pessoas, esse produto costuma ser visto como algo de gosto duvidoso e sem um significado ou função concreta.

\subsection{Produto terroir e Design}

As relações diretas entre o suvenir e o design, ou, quiçá, entre o turismo e o design, não são estabelecidas na busca bibliográfica em que consiste este artigo. Porém, é inegável a contribuição de Krucken (2009), se transportarmos a discussão que ela instala entre o design e o território para o produto terroir, mais especificamente, o suvenir como vertente desse tipo de produto. Ainda sob uma perspectiva sistêmica, 0 design pode ser aplicado a um território. Para a autora, o designer pode contribuir para fortalecer a vocação do território, desenvolvendo produtos e serviços baseados nos recursos locais e que agreguem maior valor localmente, dinamizando a economia. Para que isso ocorra, é necessário passar de uma dimensão de compreensão cultural geral a uma compreensão qualitativa e quantitativa do "sistema design".

Inserir o design na discussão proposta, significa estabelecer um diálogo com todo o teor afetivo e significativo que envolve a transformação da cultura territorial em turismo, ou seja, com a herança, a memória coletiva e a autenticidade. Para Bonsiepe (2011; 60), “a entronização da dimensão simbólica corresponde ao desprezo arrogante pela planura das funções práticas". Porém, neste aspecto, vale salientar que para esta pesquisa a dimensão simbólica tem o papel primário da função prática do suvenir estudado. Llipinar e Parkman (2011) se posicionam em relação a tensão histórica entre forma e função no design. Para os autores, este debate está centrado sobre o contraste entre o funcionalismo racional e a estética. Além de estética, design pode ser empregado para tratar de assuntos mais holísticos que podem conduzir critérios de compra, como o significado, além, da função primária de um produto.

O fato é que essas constantes discussões do design já tornaram inerente ao estudo da profissão o ato de cultivar o hábito de observar pessoas, lugares, organizações, projetos e ideias em busca de inovações que atendam as mais complexas necessidades da sociedade contemporânea. Essa tendência exige do designer a capacidade de contextualizar e globalizar, desenvolvendo soluções que favorecem os recursos e as potencialidades locais, e, simultaneamente, promover a integração das comunidades, incorporando benefícios tradicionais ou tecnológicos, e ativando diálogos e redes locais e globais.

Krucken (2009) esclarece que a crescente conscientização da importância do design para o desenvolvimento local não vem ocorrendo apenas no Brasil; e, ainda vem contribuindo para a sua integração na agenda política de diversas regiões e países. Para a continuidade desse setor, a sinergia entre profissionais com competências complementares é um elemento essencial para desenvolver projetos de caráter sistêmico. Além disso, para a autora, a habilidade para interagir com grupos 
heterogêneos é essencial na mediação e na integração de diversos universos e para a compreensão de contextos culturais plurais no projeto de produtos e serviços.

\title{
3.2 Suvenir
}

O Termo de referência: atuação do Sistema SEBRAE no artesanato categoriza nove tipos de produtos artesanais de acordo com seu processo de produção, sua origem, uso e destino (MASCÊNE at al; 2010; 12). Para esta pesquisa, vale ressaltar o que os autores deste documento entendem como suvenir:

\begin{abstract}
"Produtos semi-industriais e industriais "Industrianato/ Souvenir"
Produção em grande escala, em série, com utilização de moldes e formas, máquinas e equipamentos de reprodução, com pessoas envolvidas e conhecedoras apenas de partes do processo. Souvenirs são objetos produzidos com foco no mercado turístico, que expressam identidade cultural, comunica conceitos e busca qualidade e funcionalidade das peças."
\end{abstract}

Horodyski et al (2014) busca preceitos teóricos do turismo, da geografia humanística e da fenomenologia, como um campo filosófico, para se compreender as relações existentes entre o consumo de suvenires nas experiências dos turistas. Para os autores, outro aspecto a ser destacado, quando se trata da pouca importância dada ao suvenir, como um objeto de investigação,

\begin{abstract}
"é o fato do mesmo estar associado a fatores de descaracterização da cultura local, partindo do princípio de que muitas destas peças não são produzidas na região onde são ofertadas, embora possuam o rótulo de 'lembrança típica', concorrendo, com os artesãos da própria comunidade, ocasionando diversos aspectos danosos a cultura".
\end{abstract}

Tanto para Horodyski et al (2014) quanto para Silva (2009), suvenires são bens que representam de alguma forma o espaço visitado e complementam as experiências turísticas. Se considerarmos o suvenir como um tipo de produto terroir, Aurier, Fort e Sirieix (2005) e Llipinar e Parkman (2011) também acreditam nessa dimensão simbólica de representação espacial. O suvenir, portanto, não deve ser analisado pelo que parece ser, mas por aquilo que ele representa às pessoas, pois trata-se de um ícone ou um símbolo cultural. Fazem parte da experiência turística e são "objetos que despertam o interesse de turistas no mundo inteiro, cabe aos pesquisadores compreenderem que experiências estão envolvidas nestas motivações" (HORODYSKI et al; 2014).

Neste sentido, na busca por uma representação mais autêntica para Silva (2009), o artesanato produzido por uma comunidade local aparece como uma solução. Ainda segundo Silva (2003), essa opção fornece certa variedade e imperfeição, que, por sua vez, permite que se possa diferenciá-las e estabelecer relações simbólicas com modos de vida mais real ou com uma natureza nostálgica. A adoção de artigos artesanais no cotidiano urbano não é um fato novo, mas é importante salientar que a demanda pelo artesanal teve seu espaço bastante ampliado na contemporaneidade, "fenômeno esse que decorre da busca por diferenciação numa sociedade de produtos massificados".

No entanto, além do artesanato, Horodyski et al (2014) considera como suvenir objetos de arte, objetos industrializados, artigos alimentícios, audiovisuais, etc., "desde que representem as experiências vividas pelos turistas e as lembranças de um destino visitado". Assim, temos uma "indústria de recordações" voltada à 
consumidores na condição de turistas. O elenco teórico levantado por Horodyski et al (2014) gerou a conclusão da distinção entre esses consumidores em turistas experientes e pouco experientes em viagens, conforme se pode observar:

\begin{abstract}
"Turistas mais experientes: a autenticidade do souvenir é abstrata; o souvenir está associado a uma experiência vivida no destino turístico; o significado do souvenir consumido é pessoal; o souvenir possui representações diferentes para cada indivíduo.

Turistas menos experientes: a autenticidade é evidente; o souvenir está associado diretamente ao destino turístico; o significado do souvenir consumido é generalizado; o souvenir não possui representações, ele é aquilo pelo o que se apresenta."
\end{abstract}

A partir do exposto, turistas mais experientes "se preocupam em viver experiências autênticas e durante esses momentos adquirem um determinado produto, que muitas vezes não é comercializado em uma loja ou feira de souvenirs". De maneira oposta aos turistas com menor experiência em viagens, cuja preocupação é a comprovação de que a visita fora realizada.

Mas, embora se possa argumentar que todos os turistas da herança estão interessados em escapar da realidade atual, ou pelo menos imergirem no passado, é possível diferenciá-los entre outros dois tipos propostos por Osborne (2001): os que "fogem da realidade" e os que "fogem para a fantasia". O primeiro grupo é "altamente sensível à autenticidade percebida do objeto ou do lugar e é repelido pelo que é experimentado como patrimônio inventado". No entanto, o consumidor de" herança como fantasia é apoiado por uma categoria de "pós-turistas", que estão satisfeitos por "autenticidade encenada" e até mesmo "prazer em inautenticidade".

Quando questionada sobre o papel do suvenir durante a experiência turística, a entrevistada referente à pesquisa narrativa de Horodyski et al (2014), considerada pela autora como turista mais experiente, e compreendida sobre o ponto de vista de Osborne (2001) como turista que busca fugir da realidade, define o artefato como:

\footnotetext{
“Souvenir é outra coisa...Não é compra. É o mesmo que foto. O souvenir serve para trazer pra casa, para provocar lembranças dos passeios. Sempre compro souvenir, mas não de todos os lugares. Só dos que me emocionam. Quando estou em um lugar que me emociona, alguma coisa mexe comigo, uma vontade de conhecer tudo daquele lugar, de guardar tudo na memória, uma vontade de contar pras pessoas o que eu vivi, é uma coisa de afeto mesmo."
}

\title{
4. CONCLUSÃO
}

Foi possível concluir que os princípios potencializadores da função de representar uma experiência turística se tratam da relação combinatória entre o espaço, a cultura e a herança e/ou memória coletiva. A fonte para conhecimento desses itens está na história de um povo e um lugar; e, podem ser reconhecidas por meio de crenças, eventos, estilo arquitetônico, técnicas artesanais, receitas alimentícias, matéria-prima, etc.

Porém, resumir a complexa relação que esse estudo se propôs ao conhecimento da história não condiz com os questionamentos problematizados. Por exemplo, no tópico "3. O Produto Terroir", alguns autores foram citados por desconsiderem a industrialização como um processo de produto de terroir, assim como, no tópico "3.2 
Suvenir", o artesanato aparece como uma solução mais autêntica. Estas citações estimulam a reflexão de como podem ser anacrônicos os valores territoriais e históricos, uma vez que ao comparar uma cestaria artesanal comprada a uma índia em Manaus com um Mickey de pelúcia adquirido na Disney Word, não temos valores qualitativos de qual experiência turística é mais autêntica, e nem de qual dos suvenires é mais real.

História é o reino de fazer sentido, gradualmente, trazendo elementos comprobatórios, existe pluralidade e ambiguidade, por definição. Portanto, uma solução hipotética para este paradoxo é tentar explorar não só os "estados" originais, mas as mudanças ao longo do tempo também.

Isso significa que a autenticidade está estabelecida na comunicação subjetiva, negociada e projetada sobre o objeto e, especialmente, a cultura. Turistas lotam áreas remotas em busca de diferentes situações e estão destinados a comprar a ideia de que o objeto é genuíno e original. Esta ocasião paradoxal estimula a reflexão entre o tempo e a mudança cultural. Mesmo que a reconstrução da herança pareça ignorar o tempo, o que se discute aqui não é o processo de fabricação do suvenir oriundo de uma atividade eco turística, histórica ou de uma atração encenada; e sim, os valores subjetivos que justificam os interesses na experiência turística, e na escolha de artefatos que a represente, na crença de que serão capazes de evocar novamente as emoções vividas de uma identidade particular.

O fato é que a herança autêntica ou encenada, cada vez mais, é discutida no contexto das mitologias construídas, entretenimento popular, turismo e desenvolvimento econômico. Por isso, a iniciativa privada e todos os níveis governamentais estão reconhecendo o potencial econômico dos espaços turísticos como herança consumível (OSBORNE; 2001).

A gestão desses princípios e suas complexidades; a direção e manutenção da experiência econômica; e o sistema de produção e o produtor são premissas que tangenciam o projeto de design na atualidade. O que se defende com a conclusão deste estudo é o poder potencializador do design como recurso para a construção de uma estratégia de inovação em escala turística, imergindo-o também no universo da indústria de recordações.

\section{REFERÊNCIAS}

AURIER, P., FORT F., and SIRIEIX, L.. Exploring terroir product meanings for the consumer. Anthropology of Food, 2005.

BONSIEPE, Gui. Design, cultura e sociedade. São Paulo, Edgard Blucher, 2011.

HALL, Stuart. Identidades culturais na pós-modernidade. Trad. Por Tomaz T. da Silva e Guacira L. Louro. Rio de Janeiro: DP\&A Editora, 1997. Título original: The questiono f cultural identity.

HORODYSKI, Graziela Scalise Horodyski; MANOSSO, Franciele Cristina; GÂNDARA, José M. G. A Pesquisa Narrativa na Investigação das Experiências Turísticas Relacionadas ao Consumo de Souvenirs: uma abordagem fenomenológica. Turismo em Análise. Vol. 25, n. 1, abril 2014. 
Conceitos e Abrangência do Souvenir na Dinâmica do Espaço Turístico: O Caso

de Curitiba - PR. Revista Turismo Visão e Ação - Eletrônica, Vol. 15 - no 1 - p. 130-143 / jan-abr 2013.

KRUCKEN, Lia. Competências para o design na sociedade contemporânea. Cadernos de Estudos Avançados em Design. Belo Horizonte : Santa Clara : Centro de Estudos Teoria, Cultura e Pesquisa em Design. Caderno 2, v. 1. UEMG, 2008.

KATAN; David. Translating the tourist gaze: from heritage and 'culture' to actual encounter. PASOS. Revista de Turismo y Patrimonio Cultural. Vol. 10 No 4. Special Issue. págs. 83-95. 2012.

LENGKEEK, Jaap; The Authenticity Discourse of Heritage. Frontiers of the Roman Empire. The European Dimensiom of a World Heritage Site. 2008. Historic Scotland: Edinburg, pp. $37-53$.

LLIPINAR, Gursel; PARKMAN, Ian D. Design Culture as a Strategic orientation in new product development: a cross cultural study. International Journal of Research in Finance and Marketing, Vol. 1, No. 4, pp. 1-24, 2011.

MASCÊNE, Durcelice Cândida; TEDESCHI, Maurício. Termo de referência: atuação do Sistema SEBRAE no artesanato. Brasília: SEBRAE, 2010. Disponível em: <http://www.biblioteca.sebrae.com.br/bds/bds.nsf/4762969DAC2E2FBC8325770E005 416FC /\$File/NT00043F22.pdf> Acesso em: 12 nov. 2010.

OSBORNE, Brian S. Landscapes, Memory, Monuments, and Commemoration: Putting Identity in its Place. Academic Journal. Canadian Ethnic Studies; 2001, Vol. 33 Issue 3, p39.

PINE II, B.J.; GILMORE, J.H. The Experience Economy - work is theatre \& every business a stage. Massachusetts: Ed. Harvard Business School Press, 1999.

RENEE, Wirth; FREESTONE, Robert. Tourism, heritage and authenticity: State-assisted cultural commodification in suburban Sydney, Australia. Perspectivas Urbanas/Urban Perspectives. 2003, núm. 3.

SANTAELLA, Lúcia. Culturas e Artes do Pós-Humano. Da cultura das mídias à cibercultura. Paulus. São Paulo, 2003.

SILVA; Emanuelle K. R. da. Design e artesanato: um diferencial cultural na indústria do consumo. Actas de Diseño. Diseño en Palermo. Encuentro Latinoamericano de Diseño. Ano IV, Vol. 7, Buenos Aires, Argentina. 2009.

TERZIYSKA, Llinka. Interpretations of Authenticity in Tourism. Science\&Research, vol. 4, 2012.

WANG, N. Rethinking Authenticity in Tourism Experience. Annals of Tourism Research, V. 26, n. 2, 1999. 